\title{
Relationship between effective refractory period and inducibility of atrial fibrillation from the superior vena cava after pulmonary vein isolation
}

\author{
Yasunobu Yamagishi ${ }^{1}$, Yasushi Oginosawa ${ }^{2}$, Yoshihisa Fujino ${ }^{3}$, Keishiro Yagyu ${ }^{4}$, Taro \\ Miyamoto $^{4}$, Keita Tsukahara ${ }^{4}$, Hisaharu Ohe ${ }^{4}$, Ritsuko Kohno ${ }^{5}$, Masaharu Kataoka ${ }^{4}$, and \\ Haruhiko Abe ${ }^{6}$ \\ ${ }^{1}$ University of Occupational and Environmental Health Hospital \\ ${ }^{2}$ Sangyo Ika Daigaku \\ ${ }^{3}$ University of Occupational and Environmental Health, Japan, \\ ${ }^{4}$ University of Occupational and Environmental Health Japan \\ ${ }^{5}$ University of Occupational and Environmental Health, Kitakyushu, Japan \\ ${ }^{6}$ University of Occupational and Environmental Health, Japan
}

September 25, 2021

\begin{abstract}
Background: In terms of the pulmonary vein (PV), atrial fibrillation (AF) patients have a shorter effective refractory period (ERP) and a larger dispersion of the ERP than patients without AF. Although the frequency of AF from the superior vena cava (SVC) was the highest among non-PV foci, the characteristics of the ERP in the SVC (SVC-ERP) were unclear. The purpose of this study was to elucidate the relationship between SVC-ERP and the inducibility of AF after pulmonary vein isolation (PVI). Methods and Results: Consecutive 28 patients who underwent PVI were included. After successful PVI, the SVC-ERP was measured at three positions in SVC. Rapid electrical stimuli were delivered at the shortest SVC-ERP to induce AF. Patients in whom AF was induced were assigned to the SVC-induced group (SIG) and the remaining patients were the non-SVC-induced group (non-SIG). The size of the SVC sleeve was evaluated using three-dimensional electroanatomic mapping. The SIG had a significantly shorter average SVC-ERP (236.0 \pm 25.2 vs. $294.8 \pm 36.8 \mathrm{~ms}, \mathrm{p}<0.001)$, while SVC-ERP dispersion was not significantly different $(30.0 \pm 25.4$ vs. $33.3 \pm 20.1 \mathrm{~ms}, \mathrm{p}=0.56)$. Although the longer SVC diameter was significantly longer in the SIG (27.4 \pm 4.3 vs. $22.9 \pm 4.6 \mathrm{~mm}, \mathrm{p}=0.03)$, the SVC-ERP was significantly associated with pacing inducibility of AF after adjustment for the longer SVC diameter (odds ratio: 0.96 [1-ms increments], p=0.01). Conclusions: The SIG had a shorter SVCERP, while the dispersion was not significantly different between the two groups. The SVC-ERP can be one of the mechanisms of arrhythmogenicity for AF originating from the SVC.
\end{abstract}

\section{INTRODUCTION}

Atrial fibrillation (AF) is associated with all-cause death (especially cardiovascular death), stroke, hospitalizations, low quality of life, left ventricular dysfunction and heart failure, and cognitive decline/vascular dementia ${ }^{1}$; hence, the management of $\mathrm{AF}$ is important, and catheter ablation is an effective treatment. In 1998, Haissaguerre found that $94 \%$ of the ectopic beats that led to AF were in the pulmonary vein (PV) ${ }^{2}$, and pulmonary vein isolation (PVI) has become an established treatment for AF. However, several recent studies have revealed approximately $10-20 \%$ of AF originate from non-PV foci ${ }^{3-6}$, and the frequency of a superior vena cava (SVC) origin is the highest ${ }^{4}$. This is a major cause of reoperation. Furthermore, catheter ablation is an invasive procedure; therefore, the elimination of AF in a single procedure is desirable. However, 
SVC isolation is associated with $2.1 \%$ risk of phrenic nerve injury ${ }^{7}$ and $4.5 \%$ risk of sinus node injury ${ }^{8}$ hence, performing SVC isolation routinely in patients with AF is not recommended.

A previous seminal study reported that the effective refractory period (ERP) in patients with AF was shorter than that in patients without AF ( $185 \pm 71$ vs. $282 \pm 45 \mathrm{~ms}, P<0.001)^{9}$. This study also suggested that shorter ERPs played a major role in the development of AF. Another seminal study reported that a large dispersion of the ERP within the PVs and at the PV-left atrium (LA) junction may form a reentrant substrate and play an important role in the maintenance of $\mathrm{AF}^{10}$.

Although the frequency of an SVC origin was the highest among non-PV foci for AF, the characteristics of the ERP in the SVC (SVC-ERP) were unclear. The purpose of this study was to elucidate the relationship between the SVC-ERP and the inducibility of AF after PVI.

\section{METHODS}

\section{Study Population}

This study included 28 consecutive patients who underwent PVI with radiofrequency catheter ablation (RFCA) for paroxysmal or persistent AF at our institution. Patients with any of the following were excluded from the present study: (1) unsuccessful PVI; (2) no intrinsic atrial beat from the sinus node after PVI; and (3) no SVC sleeve.

We discontinued antiarrhythmic drugs for at least five half-lives prior to the ablation procedure to the extent that they had no adverse clinical effects, anticoagulant agents were administered at least three weeks prior to the ablation procedure, and the absence of a thrombus in the LA was confirmed by transoesophageal echocardiography. Verbal and written informed consent for this study was obtained from all patients prior to the procedure. The study protocol, including data collection and record keeping, was approved by the Institutional Review Board of the University of Occupational and Environmental Health, Japan (approval number: UOEHCRB20-035) ${ }^{11}$.

\section{PVI Procedure}

The procedure was performed under sedation with intravenous dexmedetomidine. A temperature probe (SensiThermTM; St. Jude Medical) for monitoring the oesophageal temperature was inserted via the nasal fossa and placed between the level of the superior and inferior pulmonary veins. Arterial access was routinely acquired for continuous blood pressure. A total of 3,000 units of heparin were administered after both arterial and venous access was obtained. A total of 2,000 units of heparin were administered following transseptal puncture. Heparinised saline was also infused to maintain an activated clotting time of 300-350 seconds. Ablation catheters were inserted from the right atrium (RA) to the LA via the atrial septum. Under the guidance of three-dimensional electroanatomic mapping (CARTO 3 version 7.0; Biosense Webster, Inc., California, USA), RF energy of 25-30 W was applied using a quadripolar 3.5-mm-tip irrigation catheter (Thermocool, Biosense Webster, Diamond Bar, CA, USA). The target ablation index was 400 in the right PVs and anterior of the left PVs and 350 in the posterior of the left PVs, except for the perioesophageal. After the successful achievement of bidirectional conduction block between the PVs and LA, PVI was reconfirmed by waiting for at least 30 minutes of the observational period.

\section{Identification of the SVC-RA junction and measurement of the SVC sleeve}

After the complete PVI procedure, the voltage and activation maps of the SVC and RA were precisely reconstructed using the CARTO system with a 20-pole PentaRayß)catheter (Biosense Webster, Diamond Bar, CA, USA) during sinus rhythm. The SVC-RA junction was defined as fused SVC potentials and RA potentials ${ }^{12,13}$. The cut-off value of the voltage map was $0.5-0.1 \mathrm{mV}$. Less than $0.1 \mathrm{mV}$ was defined as no electric potential (in the SVC, it was defined as no SVC sleeve). Then, the length from the SVC-RA junction to the top of the myocardial sleeve in the SVC was measured. The long and short diameters of the SVC at the height of the SVC-RA junction were also measured.

\section{Evaluation of the SVC-ERP}


The ablation catheter was used to pace the anterior, septal, and posterior sides $2 \mathrm{~mm}$ above the SVC-RA junction (Figure 1). The lateral side was not paced because of the common site of phrenic nerve stimulation. We used a stimulator (SEC-5104; Nihon Kohden, Tokyo, Japan) to deliver electrical impulses of 2-ms duration at twice the diastolic pacing threshold. The distal electrode of the catheter was the negative pole. Stable pacing sites were considered only if the output threshold was less than $4 \mathrm{~V}$ under the contact force range from 5 to $20 \mathrm{~g}$. Surface and intracardiac electrograms filtered at 50-300 Hz were recorded simultaneously using a polygraph (RMC-5000; Nihon Kohden, Tokyo, Japan). After eight stimuli at a basic cycle length of $600 \mathrm{~ms}$, a single extrastimuli coupled at $400 \mathrm{~ms}$ was decremented automatically in steps of $20 \mathrm{~ms}$ to the ERP ${ }^{9}$. The dispersion of the SVC-ERP was defined as the difference between the longest SVC-ERP and the shortest SVC-ERP.

\section{Protocol of rapid electrical stimuli from the SVC}

Rapid electrical stimuli were delivered at a portion of the shortest SVC-ERP to induce AF. Pacing with a 2-ms duration and three times the voltage of the threshold was delivered as 40 beats of pacing with an initial pacing cycle length (PCL) of $500 \mathrm{~ms}$, which was shortened by 50 -ms increments down to $250 \mathrm{~ms}$, after which the PCL was shortened by $10-\mathrm{ms}$ increments from $250 \mathrm{~ms}$ to $160 \mathrm{~ms}{ }^{13}$. In the case of a Wenckebach conduction block or two-to-one SVC-RA conduction block as the PCL was shortened without AF induction, these groups were assigned to the non-SVC-induced group (non-SIG). If AF was induced (Figure 2), these groups were assigned to the SVC-induced group (SIG). SVC isolation was performed only in patients with reproducible induction of AF from the SVC using adenosine triphosphate or isoproterenol, and there was a sufficient margin of at least $1 \mathrm{~cm}$ from the sinus node to the isolation line.

\section{Statistical analysis}

We used the Mann-Whitney U test to compare the differences between the two independent groups when the dependent variable was either ordinal or continuous, but not normally distributed. Multivariable logistic regression models were used to estimate the relationship among the average SVC-ERP, longer SVC diameter, and pacing inducibility of AF. All statistical analyses were conducted using Stata (version 16.1; StataCorp LLC, College Station, TX, USA).

\section{RESULTS}

Of the 40 consecutive patients, who underwent PVI with RFCA for paroxysmal or persistent AF in our institution between October 2020 and July 2021, we excluded cases with withdrawal of informed consent $(\mathrm{n}=3)$, inability to keep the patient at rest due to prolonged procedure time $(\mathrm{n}=1)$, unsuccessful PVI $(\mathrm{n}=1)$, no intrinsic atrial beat from sinus node after PVI $(n=2)$, or no SVC sleeve $(n=5)$.

\section{Clinical characteristics and echocardiographic parameters}

The present study included 28 consecutive patients (age, 69.5 \pm 9.2 year; 22 (79\%) were male patients). The patients were divided into two groups. The SIG consisted of $10(36 \%)$ patients, and the non-SIG consisted of $18(64 \%)$ patients. The baseline characteristics and echocardiographic parameters of the two groups are shown in Table 1. The SIG was associated with a higher proportion of patients with persistent AF compared to the non-SIG.

\section{Electrophysiological and electrical parameters}

Overall, 28 SVCs were studied at 84 pacing sites. There was no significant difference in the threshold (average threshold was $1.6 \pm 0.5 \mathrm{~V}$ in the SIG vs. $1.6 \pm 0.5 \mathrm{~V}$ in the non-SIG, at 2-ms pulse duration, $\mathrm{p}=0.61$ ), the dispersion of the SVC-ERP (30.0 $\pm 25.4 \mathrm{~ms}$ in the SIG vs. $33.3 \pm 20.1 \mathrm{~ms}$ in the non-SIG, $\mathrm{p}=0.56)$, length of the SVC $(29.6 \pm 9.0 \mathrm{~mm}$ in the SIG vs. $27.8 \pm 11.2 \mathrm{~mm}$ in the non-SIG, $\mathrm{p}=0.70)$, and shorter diameter of the SVC $(16.9 \pm 3.3 \mathrm{~mm}$ in the SIG vs. $15.6 \pm 3.3 \mathrm{~mm}$ in the non-SIG, $\mathrm{p}=0.30)$ between both groups. However, SVC-ERPs were significantly shorter (average SVC-ERP was $236.0 \pm 25.2$ vs. $294.8 \pm 36.8 \mathrm{~ms}, \mathrm{p}<0.001$ ) and longer diameters of the SVC sleeve were significantly longer $(27.4 \pm 4.3$ vs. $22.9 \pm 4.6 \mathrm{~mm}, \mathrm{p}=0.03)$ in the SIG than in the non-SIG (Table 2). 


\section{DISCUSSION}

We investigated the relationship between the characteristics of the SVC-ERP and the pacing inducibility of AF. Our major findings were as follows: (1) the mean value of the average SVC-ERP in all patients was $273.8 \pm 43.4 \mathrm{~ms}$; (2) the pacing-induced AF rate was $36 \%$; (3) the SIG had a significantly shorter SVC-ERP than the non-SIG; and (4) the SVC-ERP dispersion was not significantly different between the two groups.

\section{SVC-ERP in humans}

Although SVC-ERP measurements in sheep ${ }^{14}$ and $\operatorname{dogs}{ }^{15}$ have been reported previously, to the best of our knowledge, there are very few reports that have examined the SVC-ERP in humans in detail. During the course of our study, we could only find one relevant study ${ }^{16}$. They investigated 20 patients with paroxysmal supraventricular tachycardia and reported that the SVC-ERP conducted by a basic drive cycle of eight stimuli at a cycle length of 600 was $266 \pm 17.3$. However, the SVC-ERP in all AF patients in our study was $280.0 \pm 49.0$ in the anterior, $269.2 \pm 46.0$ in the septal, $272.1 \pm 45.0$ in the posterior parts, and the average SVC$\mathrm{ERP}$ was $273.8 \pm 43.4 \mathrm{~ms}$. The mean dispersion of the SVC-ERP was $32.1 \pm 22.0$. Since there may be several factors that can modify the SVC-ERP (e.g. electrical remodelling, ganglionated plexi, and antiarrhythmic drugs), the measurement of the SVC-ERP in healthy subjects must be included as a control.

\section{Pacing inducibility from the SVC}

We performed rapid electrical stimulation from the SVC to induce AF based on previously published research

${ }^{13}$, but the induction rate was much higher ( $36 \%$ in our study vs. $19 \%$ in a previous study). The possible reason was that in the previous study, they only paced at one point in the SVC and did not investigate the SVC-ERP, whereas we investigated the three portions of the SVC and performed burst pacing from the shortest SVC-ERP. Therefore, the induction rate in the present study was considered to be high.

\section{Factors related to AF originating from the SVC}

Several previous studies have elucidated the relationship between some factors and AF originating from the SVC (SVC-AF). Almost all of these studies used pacing and/or drugs to induce SVC-AF. An anatomical report showed that SVC-AF is more likely to occur in patients with a smaller LA diameter ${ }^{17}$. In a report on clinical features, SVC-AF tends to be more common in female patients, those with a lower body mass index, and those with certain genes (rs2634073 and rs6584555) ${ }^{18}$. The electrical characteristics were that SVC-AF is more common in patients with long SVC sleeves $\left(>30 \mathrm{~mm}^{12,13}\right)$, SVC firing is more likely to occur in patients with long SVC sleeves $(>37 \mathrm{~mm})$ or long SVC diameters $(>17 \mathrm{~mm})^{19}$, and $\mathrm{SVC}-\mathrm{AF}$ is more common in cases with a large potential $\left(>1 \mathrm{mv}^{12}\right)$.

In our electrophysiological study, the relationship between the SVC-ERP and SVC-AF was clarified. To the best of our knowledge, there are no reports that have examined the relationship between the SVC-ERP and SVC-AF. Our study revealed that the shorter the SVC-ERP, the more likely SVC-AF occurred at pacing, while the dispersion of the SVC-ERP did not contribute.

The present study revealed that the SIG had a higher proportion of patients with persistent AF. Past animal experiments using goats reported that $\mathrm{AF}$ leads to a marked shortening of the atrial $\mathrm{ERP}^{20}$, and this electrophysiological change is called electrical remodelling. Another previous study reported that electrical remodelling may also occur in the human SVC ${ }^{16}$. Our study revealed the concordant result; patient with persistent AF had a shorter SVC-ERP than those with paroxysmal AF (240.0 \pm 37.6 vs $295.7 \pm 31.7 \mathrm{~ms}$, $\mathrm{p}=0.002$ ). We assumed that persistent AF caused the SVC sleeve to have a stronger electrical remodelling than that caused by paroxysmal AF, resulting in the acquisition of a shorter SVC-ERP and a greater vulnerability to pacing from the SVC.

Although previous studies using the same rapid electrical stimuli reported that the length of the SVC sleeve was longer in the $\mathrm{SIG}^{13}$, we did not find a difference between the two groups. The possible reason was that we selected only patients who could measure the SVC-ERP in all three portions. As a result of selecting a 
population with a relatively long SVC sleeve length, there may be no difference between the two groups in the present study.

A previous study reported that SVC firing is more likely to occur in patients with long SVC sleeves $(>37$ $\mathrm{mm})$ or long SVC diameters $(>17 \mathrm{~mm})^{19}$. Our study also revealed that the longer diameter of the SVC was significantly longer in the SIG $(27.4 \pm 4.3$ vs. $22.9 \pm 4.6 \mathrm{~mm}, \mathrm{p}=0.03)$ compared to that of the non-SIG. Furthermore, we found that the SVC-ERP was significantly associated with pacing inducibility of AF after adjustment for the longer diameter of the SVC (adjusted odds ratio: 0.96 [1-ms increments], 95\% confidence interval: $0.93-0.99 ; \mathrm{p}=0.01)$. It was suggested that the SVC-ERP was also a factor related to SVC-AF.

\section{Clinical implications}

In the present study, we investigated the SVC-ERP and inducibility of SVC-AF after PVI because the frequency of SVC origin was the highest among non-PV foci for AF. We revealed that the SVC-ERP was significantly associated with pacing inducibility of AF after adjusting for a longer SVC diameter. It was suggested that the measurement of the SVC-ERP may predict SVC-AF. Furthermore, in our study, the dispersion of the SVC-ERP did not contribute to arrhythmogenicity at least just above the SVC-RA junction. Only the shortest SVC-ERP may be an indicator of SVC-AF inducibility.

\section{Limitations}

This study has several limitations. First, the study population was small; hence, our results should be confirmed in a large-scale study. Second, we measured the dispersion of the SVC-ERP only on the same plane. Kumagai et al . measured the ERP in both distal PVs and the PV-LA junction ${ }^{10}$ and found the dispersion of the ERP within PVs. Finally, because only pacing inducibility was assessed in the present study, future studies on the relationship between the SVC-ERP and spontaneously occurring SVC-AF are desirable.

\section{CONCLUSIONS}

Our study revealed that the SIG had a shorter SVC-ERP than the non-SIG; however, the dispersion of the SVC-ERP did not contribute. Because a shorter SVC-ERP was associated with pacing inducibility of AF, the SVC-ERP can be one of the mechanisms of arrhythmogenicity in cases of SVC-AF.

\section{COMPETING INTERESTS}

The authors have no competing interests.

\section{FUNDING}

This research received no specific grant from any funding agency in the public, commercial, or not-for-profit sectors.

\section{AUTHORS' CONTRIBUTIONS}

YY was involved in data analysis and writing of the manuscript. YO was involved in data verification, study design, supervision, and manuscript revision. YF was involved in data verification, supervision, and statistical analyses. KY, TM, and KT were used for the data verification. HO and RK were involved in the data verification and supervision. MK and HA were involved in data verification, supervision, and manuscript revision.

\section{References}

1. Kirchhof P, Benussi S, Kotecha D, et al. 2016 ESC Guidelines for the management of atrial fibrillation developed in collaboration with EACTS. Eur Heart J. 2016;37(38):2893-2962.

2. Haissaguerre M, Jais P, Shah DC, et al. Spontaneous initiation of atrial fibrillation by ectopic beats originating in the pulmonary veins. $N$ Engl J Med. 1998;339(10):659-666. 
3. Bhargava M, Di Biase L, Mohanty P, et al. Impact of type of atrial fibrillation and repeat catheter ablation on long-term freedom from atrial fibrillation: results from a multicenter study. Heart Rhythm. 2009;6(10):1403-1412.

4. Hayashi K, An Y, Nagashima M, et al. Importance of nonpulmonary vein foci in catheter ablation for paroxysmal atrial fibrillation.Heart Rhythm. 2015;12(9):1918-1924.

5. Hung Y, Lo LW, Lin YJ, et al. Characteristics and long-term catheter ablation outcome in long-standing persistent atrial fibrillation patients with non-pulmonary vein triggers. Int J Cardiol.2017;241:205-211.

6. Santangeli P, Zado ES, Hutchinson MD, et al. Prevalence and distribution of focal triggers in persistent and long-standing persistent atrial fibrillation. Heart Rhythm. 2016;13(2):374-382.

7. Miyazaki S, Usui E, Kusa S, et al. Prevalence and clinical outcome of phrenic nerve injury during superior vena cava isolation and circumferential pulmonary vein antrum isolation using radiofrequency energy. $\mathrm{Am}$ Heart J. 2014;168(6):846-853.

8. Chen G, Dong JZ, Liu XP, et al. Sinus node injury as a result of superior vena cava isolation during catheter ablation for atrial fibrillation and atrial flutter. Pacing Clin Electrophysiol.2011;34(2):163-170.

9. Jais P, Hocini M, Macle L, et al. Distinctive electrophysiological properties of pulmonary veins in patients with atrial fibrillation. Circulation. 2002;106(19):2479-2485.

10. Kumagai K, Ogawa M, Noguchi H, Yasuda T, Nakashima H, Saku K. Electrophysiologic properties of pulmonary veins assessed using a multielectrode basket catheter. J Am Coll Cardiol.2004;43(12):2281-2289.

11. University of Occupational and Environmental Health, Japan.,. Ethics Committee of Medical Research,University of Occupational and Environmental Health,Japan.https://www.uoehu.ac.jp/industryCo/kenkyu/top.html. Accessed 16 May, 2021.

12. Higuchi K, Yamauchi Y, Hirao K, et al. Superior vena cava as initiator of atrial fibrillation: factors related to its arrhythmogenicity. Heart Rhythm. 2010;7(9):1186-1191.

13. Nakamura T, Hachiya H, Yagishita A, et al. The Relationship between the Profiles of SVC and Sustainability of SVC Fibrillation Induced by Provocative Electrical Stimulation. Pacing and clinical electrophysiology : PACE. 2016;39(4):352-360.

14. Zhao C, Qi J, Liu X, et al. Refractoriness of the sheep superior vena cava myocardial sleeve. Exp Biol Med (Maywood).2008;233(11):1441-1447.

15. Lee SH, Chen YJ, Tai CT, et al. Electrical remodeling of the canine superior vena cava after chronic rapid atrial pacing. Basic Res Cardiol. 2005;100(1):14-21.

16. Lee KT, Chu CS, Lin TH, et al. Effects of verapamil on superior vena cava electrical remodeling induced by short-term pacing from right atrium and superior vena cava in human. Int J Cardiol.2007;120(3):380-386.

17. Miyazaki S, Taniguchi H, Kusa S, et al. Factors predicting an arrhythmogenic superior vena cava in atrial fibrillation ablation: insight into the mechanism. Heart Rhythm. 2014;11(9):1560-1566.

18. Ebana Y, Nitta J, Takahashi Y, et al. Association of the Clinical and Genetic Factors With Superior Vena Cava Arrhythmogenicity in Atrial Fibrillation. Circ J. 2017;82(1):71-77.

19. Nyuta E, Takemoto M, Sakai T, et al. Importance of the length of the myocardial sleeve in the superior vena cava in patients with atrial fibrillation. Journal of Arrhythmia. 2021;00(n/a):1-9.

20. Wijffels MC, Kirchhof CJ, Dorland R, Allessie MA. Atrial fibrillation begets atrial fibrillation. A study in awake chronically instrumented goats. Circulation. 1995;92(7):1954-1968.

Figure Legends

Figure 1. Evaluation of ERP in the three portions of the SVC 
The left-side figure (A) shows the superior view, and the right-side figure (B) shows the left anterior oblique view in CARTO. Orange tags were defined as the anterior, septal, and posterior sides, $2 \mathrm{~mm}$ above the SVC-RA junction. An ablation catheter was used to pace these three portions to investigate the SVC-ERP. The lateral side was not paced because of the common site of phrenic nerve stimulation. The length from the SVC-RA junction to the top of the myocardial sleeve and the longer and shorter diameters of the SVC at the height of the SVC-RA junction were also measured

Abbreviations: ERP, effective refractory period; RA, right atrium; SVC, superior vena cava.

\section{Figure 2. Induction of SVC-AF}

AF was induced by $170 \mathrm{~ms}$ of rapid electrical stimuli from the portion of the shortest SVC-ERP (ABL 1-2). The red asterisks indicate the initiation of SVC-AF from the SVC potential. The Pentaray catheter was within the SVC, and SVC potentials were recorded.

Abbreviations: ABL, ablation site; AF, atrial fibrillation; CS, coronary sinus; ERP, effective refractory period; HRA, high right atrium; RVa, right ventricular apex; SVC, superior vena cava.

Tables

Table 1. Clinical characteristics and echocardiographic parameters Variables

Age, years, mean (SD)

Sex, men proportion, $\%$

BMI, $\mathrm{kg} / \mathrm{m}^{2}$, mean (SD)

Paroxysmal AF/Persistent AF \%

LA size, mm, mean (SD)

LVEF, \%, mean (SD)

Medication (Vaughan-Williams classification)

None

ClassI

ClassII

ClassIII

ClassIV

SIG was associated with a higher proportion of patients with persistent AF. Other parameters were not significantly differe

Table 2. Electrophysiological and electrical parameters

Parameter

Threshold in SVC, V at 2ms, mean (SD)

Anterior

Septal

Posterior

Average

SVC-ERP, ms, mean (SD)

Anterior

Septal

Posterior

Average

Dispersion

SVC sleeve, mm, mean (SD)

Length of SVC

Longer diameter of SVC

Shorter diameter of SVC 
The SVC-ERP was significantly shorter and longer SVC diameters were significantly longer in the SIG than in the non-SIG
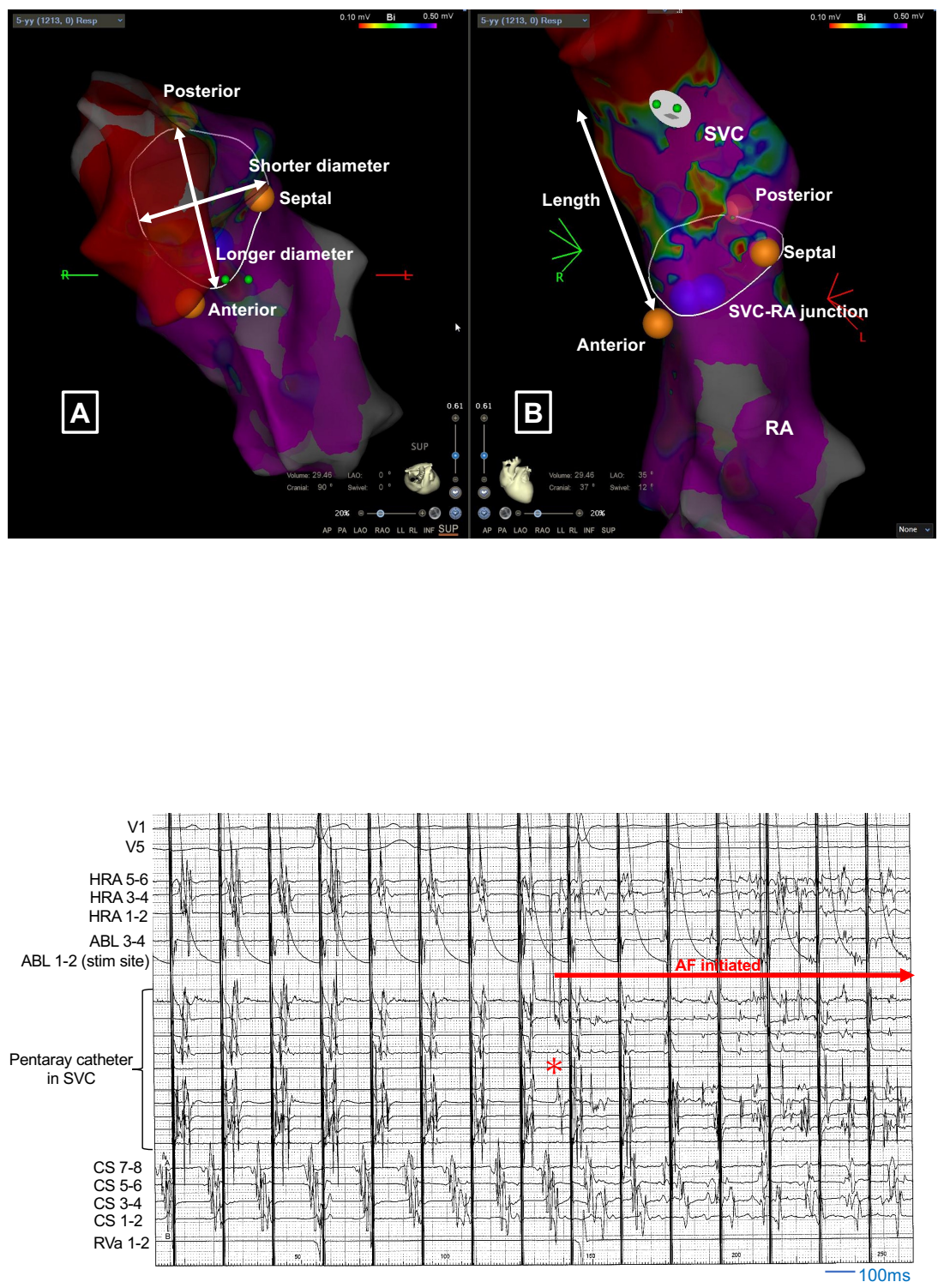